\title{
Standard quasi-conformal flattening of the right and left atria
}

\author{
Marta Nuñez-Garcia ${ }^{1,2}$, Gabriel Bernardino ${ }^{1,3}$, Ruben Doste $^{1}$, Jichao Zhao ${ }^{4}$, \\ Oscar Camara ${ }^{1}$, and Constantine Butakoff ${ }^{1}$ \\ 1 Physense, Department of Information and Communication Technologies, \\ Universitat Pompeu Fabra, Barcelona, Spain \\ ${ }^{2}$ L'Institut de Rythmologie et de Modélisation Cardiaque LIRYC/Université de \\ Bordeaux, France \\ 3 Philips Research, Medisys, France \\ 4 Auckland Bioengineering Institute, University of Auckland, New Zealand
}

\begin{abstract}
Two-dimensional standard representations of 3D anatomical structures are a simple and intuitive way for analysing patient information across populations and image modalities. They also allow convenient visualizations that can be included in clinical reports for a fast overview of the whole structure. While cardiac ventricles, especially the left ventricle, have an established standard representation (e.g. bull's eye plot), the $2 \mathrm{D}$ depiction of the left (LA) and right atrium (RA) remains challenging due to their sub-structural complexity. Quasi-conformal flattening techniques, successfully applied to cardiac ventricles, require additional constraints in the case of the atria to correctly place the adjacent structures, i.e. the pulmonary veins, the vena cava (VC) or the appendages. Some registration-based methods exist to flatten the LA but they can be timeconsuming and prone to errors if the geometries are very different. We propose a novel atrial flattening methodology where a quasi-conformal 2D map of both (left and right) atria is obtained quickly and without errors related to registration. In our approach the RA is mapped to a standard 2D map where the holes corresponding to superior and inferior VC are fixed within a disk. Similarly, the LA is divided into 5 regions which are then mapped to their analogous two-dimensional regions. We illustrate the application of the method to visualize atrial wall thickness measurements, and late gadolinium enhanced magnetic resonance data.
\end{abstract}

Keywords: Conformal flattening · two-dimensional map · left atrium . right atrium $\cdot$ atrial wall thickness $\cdot$ LGE-CMR

\section{Introduction}

Flattening methods aim to compute an unfolded representation of a 3D surface mesh by projecting it to a simpler $2 \mathrm{D}$ domain, easier to visualize, manage and interpret. Additionally, if the 2D map is standardised (e.g. same anatomical regions of different subjects spatially coincide) the $2 \mathrm{D}$ unfolded domains can be used as a common reference space to analyse multi-modal data from different 
patients or from the same patient at different time-steps. The reader is referred to [5] for a thorough review of flattening methods applied to human organs, including the brain, different bones, and the vascular system.

In the case of the heart, the 17 segment AHA bull's eye plot of the left ventricle (LV) has been widely used by clinicians for long time $[2,15,11]$. The LV's conical shape and the absence of salient sub-structures in 3D anatomies derived from medical images (e.g. ignoring the trabeculations) highly facilitates its flattening. On the contrary, unfolding the atria is challenging due to their more complex morphology. In the case of the LA, its main cavity is connected to several pulmonary veins (PV), the left atrial appendage (LAA) and the LV through the mitral valve (MV). Furthermore, the most common LA morphology (up to $70 \%$ ) involves 4 PV (with variable size, shape, position and orientation with respect to the main cavity) but in some cases a common left trunk, extra right PV or other oddities may be present [13]. Something similar occurs in the case of the RA, which is connected to the right ventricle through the tricuspid valve (TV), to the superior and inferior vena cava (SVC and IVC), and to the coronary veins $(\mathrm{CV})$.

The first LA flattening technique available in the literature [8] was based on a B-spline with proportional distance between any pair of points in the 3D LA surface and their mapped pairs on the 2D map. Later, the same group proposed to flatten the LA to a square also without constraining the position of the PV and LAA holes [3]. The authors showed how the LA flattened square could be used to display and qualitatively analyse different types of data from the same patient. However, a direct comparison across different patients was not feasible because of the lack of correspondence between the different maps. More recently, Williams et al. [17] proposed a standardised unfold map (SUM), which flattening strategy was based on registering an arbitrary LA to a $3 \mathrm{D}$ template and then transferring the data to a 2D LA template using the known 3D-2D point relation between the two templates. The main limitation of this approach is the difficulty of obtaining an accurate and fast registration between different LA surface meshes due to their high shape variability. Together with errors induced by required data projection and interpolation steps, this scheme leads to undesired information loss between the 3D and 2D LA representations. Recently, a universal atrial coordinate system was developed in [14] and used to represent both atria in a two-dimensional domain. The proposed representation is however not completely standardised since the position of the veins and appendages may be distinct for the different cases.

In this paper, we propose a method to represent atrial cavities as $2 \mathrm{D}$ disks where the position of the holes corresponding to the different veins and appendages is fixed. Our method is almost real-time and without information loss, i.e. all points in the $3 \mathrm{D}$ mesh are represented in the $2 \mathrm{D}$ domain. 


\section{Methodology}

The proposed method is based on a quasi-conformal (in general, there is no conformal map compatible with a given map along the boundary) flattening parameterisation [6]. The RA is unfolded with the following boundary constraints: the TV contour is mapped to the external circumference of a $2 \mathrm{D}$ disk, and the holes corresponding to the SVC and IVC are mapped to predefined circumferences within the disk. Additionally, one constraint is added to fix the position of the right atrial appendage (RAA). Regarding the LA, the MV contour is also mapped to the external circumference of a 2D disk, and the PVs and LAA ostia contours are mapped to predefined circumferences within the disk. Due to the fact that flattening of surface meshes with holes often results in undesired mesh self-folding (holes appear covered by adjacent mesh cells), we impose, only for the LA, additional regional constraints: five anatomical regions are defined in the 3D LA which are afterwards confined to their 2D counterparts.

It was experimentally found that the use of additional constraints was not necessary in the case of the RA because mesh self-folding was not found in the $2 \mathrm{D}$ maps due to the intrinsically simpler RA shape (as well as the defined 2D RA template), with, for example, less boundaries compared to the LA. Another difference is that we decided to include the RAA (while not including the LAA) in the $2 \mathrm{D}$ representation, and constrain only one point since the boundary between the main body of the RA and RAA is unclear and not as well defined as in the case of the LAA, where a prominent ostium is typically present.

Given a RA or LA with holes corresponding to the valves (TV, MV) and to the connected sub-structures (SVC, IVC, PVs, LAA) the pipeline comprises two main steps: (1) definition of boundary and regional constraints; and (2) regional flattening (see Fig. 1).

\subsection{Definition of boundary and regional constraints}

Holes in the main atrial cavities are firstly closed and triangulated as in [7]. Then, seed points are manually placed in specific atrial regions:

1. Right atrium: two seed points in the filled SVC and IVC holes, and one seed point in the most salient point of the RAA.

2. Left atrium: Five seeds at the centre of the filled PV and LAA holes, and 4 on the MV contour: 2 delimiting the interatrial septal wall and 2 delimiting the left lateral wall. Inter-seed geodesic paths (i.e. shortest curve between two points on a mesh such that the curve lies on the surface [9], $\mathrm{s}_{1}-\mathrm{s}_{9}$ in Fig. 1) are computed dividing the LA surface into 5 regions.

After that, the final atrial cavities that will be flattened are obtained by automatically removing the hole covers. The boundary constrained points (mesh vertices of the red curves in Fig. 1) are identified as the SVC, ICV and TV boundary points in the case of the RA, and as the PV, LAA and MV boundary points in the case of the LA. Additionally and only for the LA, the regional 


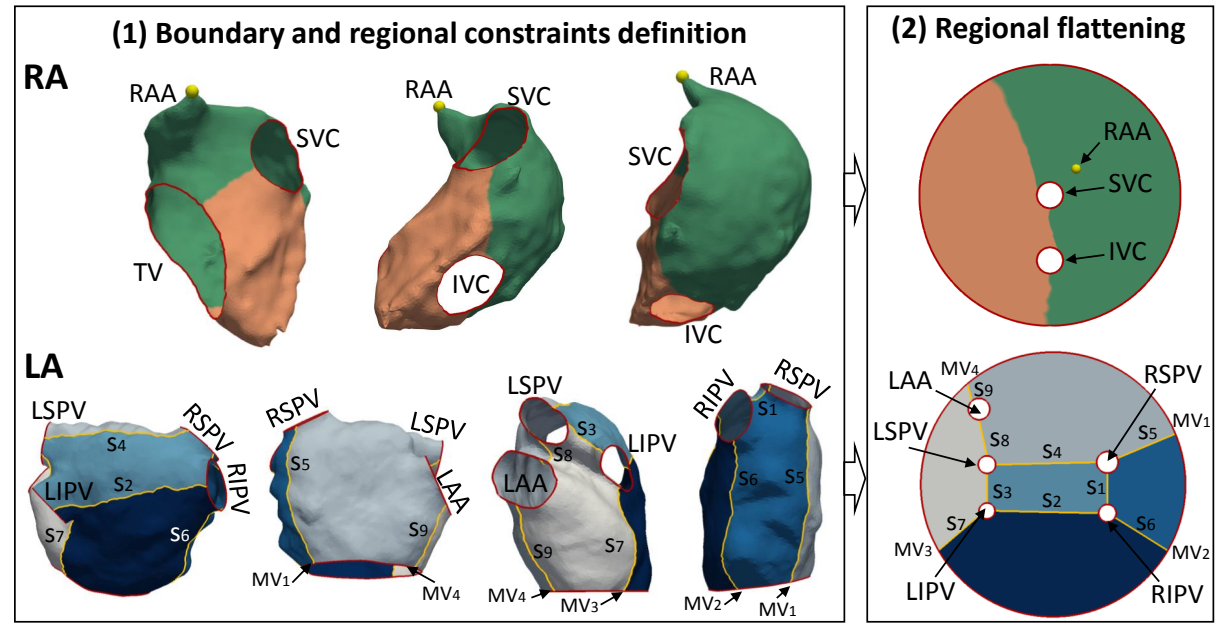

Fig. 1. Pipeline of the proposed RA (top) and LA (bottom) flattening method: (1) definition of boundary and regional constraints: nine boundaries (displayed in red) corresponding to the TV, IVC, SVC, MV, $4 \mathrm{PV}$, and LAA ostia, and nine segment paths ( $\mathrm{s}_{1-9}$, in yellow) are used. (2) Regional flattening. Data mapped into the surface meshes correspond to intensity values from the associated late-gadolinium magnetic resonance image. $\mathrm{LAA} / \mathrm{RAA}=$ left/right atrial appendage; $\mathrm{TV}=$ tricuspid valve; $\mathrm{SVC} / \mathrm{IVC}=$ superior/inferior vena cava; LSPV = left superior PV; LIPV = left inferior PV; RSPV $=$ right superior $\mathrm{PV} ; \mathrm{RIPV}=$ right inferior $\mathrm{PV} ; \mathrm{MV}=$ mitral valve; $\mathrm{MV}_{1,2}=$ seed points in the MV contour delimiting the septal wall, and $\mathrm{MV}_{3,4}=$ seed points in the $\mathrm{MV}$ contour delimiting the left lateral wall.

constrained points (points of the yellow curves in Fig. 1) are identified as the projection of the inter-seed paths $\left(\mathrm{s}_{1}-\mathrm{s}_{9}\right)$ onto the final LA cavity. Note that for the RA we only consider one regional constrained point, the furthest point in the RAA (also shown in yellow in Fig. 1).

\subsection{Regional Flattening}

With the aim of obtaining a quasi-conformal (i.e. angle-preserving) and standardised flat representation of a 3D atrial surface mesh whose holes are constrained to predefined circumferences within a 2D disk, the corresponding boundary constraints can be added to the quasi-conformal scheme. Unfortunately, the flattened holes will appear often covered by adjacent triangles since the method does not avoid mesh self-folding. To overcome this issue, we include additional regional constraints in the parameterisation. Minor triangle overlapping can still occur near the holes and to further refine the boundary we recompute the point coordinates on the 2D RA or LA with a quasi-conformal parameterisation only constrained with the boundary points and not the regional constraints. 
Mathematical framework Let $M$ be the atrial cavity surface mesh with $\mathrm{N}$ vertices (points) and $M_{F}$ its corresponding flattened mesh. Let $I_{\partial M}$ be the indices of the boundary points of $M$. Let $\Delta_{M}$ be the $N \times N$ (cotangent [12]) Laplacian of $M$ and let $\Delta_{M}^{\prime}$ be $\Delta_{M}$ where the off-diagonal elements of the rows defined by the positions of $I_{\partial M}$ set to 0 and corresponding elements on the main diagonal set to 1 . Let $\left(b_{x}, b_{y}\right)$ be the coordinates of the $2 \mathrm{D}$ boundary points of $M_{F}$ in the same order as the corresponding indices appear in $I_{\partial M}$ and let $\boldsymbol{b}_{x}^{\prime}$ and $\boldsymbol{b}_{y}^{\prime}$ be N-dimensional vectors with values $\boldsymbol{b}_{x}$ and $\boldsymbol{b}_{y}$, respectively, in the positions of $I_{\partial M}$ and zeros elsewhere.

Let $\mathrm{P}$ be the number of regional constrained points (i.e. number of points in the dividing segments $\left(\mathrm{s}_{1}-\mathrm{s}_{9}\right)$, yellow lines in Fig. 1) and $I_{s}$ the corresponding point (vertex) indices. Let $\left(s_{x}, s_{y}\right)$ be the $2 \mathrm{D}$ coordinates of the $\mathrm{P}$ vertices in the same order as they appear in $I_{s}$ and let $\boldsymbol{E}_{s}$ be a $P \times N$ zero matrix with 1 in each row in the positions corresponding to vertices of $I_{s}$ (i-th row has 1 in the position given by the i-th element of $I_{s}$ ).

In order to find the coordinates $\left(\boldsymbol{x}^{*}, \boldsymbol{y}^{*}\right)$ of the vertices of $M_{F}$, we propose to solve the following two quadratic programming problems:

$$
\begin{array}{lll}
\boldsymbol{x}^{*}=\arg \min _{\boldsymbol{x}}\left(w\left\|\boldsymbol{\Delta}_{M}^{\prime} \boldsymbol{x}-\boldsymbol{b}_{x}^{\prime}\right\|^{2}\right) & \text { s.t. } & \boldsymbol{E}_{s} \boldsymbol{x}=\boldsymbol{s}_{x} \\
\boldsymbol{y}^{*}=\arg \min _{\boldsymbol{y}}\left(w\left\|\boldsymbol{\Delta}_{M}^{\prime} \boldsymbol{y}-\boldsymbol{b}_{y}^{\prime}\right\|^{2}\right) & \text { s.t. } & \boldsymbol{E}_{s} \boldsymbol{y}=\boldsymbol{s}_{y}
\end{array}
$$

with $w$ (set to 1000 in our experiments) penalizing the unfulfillment of the boundary constraints. Using Lagrange multipliers these can be rewritten and solved as a system of linear equations:

$$
\begin{aligned}
& {\left[\begin{array}{cc}
w^{2} \boldsymbol{\Delta}_{M}^{\prime T} \boldsymbol{\Delta}_{M}^{\prime} & \boldsymbol{E}_{s}^{T} \\
\boldsymbol{E}_{s} & \mathbf{0}
\end{array}\right]\left[\begin{array}{c}
\boldsymbol{x}^{*} \\
\boldsymbol{\lambda}_{x}
\end{array}\right]=\left[\begin{array}{c}
w^{2} \boldsymbol{\Delta}_{M}^{\prime T} \boldsymbol{b}_{x}^{\prime} \\
\boldsymbol{s}_{x}
\end{array}\right]} \\
& {\left[\begin{array}{cc}
w^{2} \boldsymbol{\Delta}_{M}^{\prime} \boldsymbol{\Delta}_{M}^{\prime} & \boldsymbol{E}_{s}^{T} \\
\boldsymbol{E}_{s} & \mathbf{0}
\end{array}\right]\left[\begin{array}{c}
\boldsymbol{y}^{*} \\
\boldsymbol{\lambda}_{y}
\end{array}\right]=\left[\begin{array}{c}
w^{2} \boldsymbol{\Delta}_{M}^{\prime T} \boldsymbol{b}_{y}^{\prime} \\
\boldsymbol{s}_{y}
\end{array}\right]}
\end{aligned}
$$

To refine the boundary we proceed as follows. Let $\boldsymbol{\Delta}_{F}$ be the $N \times N$ Laplacian of $M_{F}$ and let $\Delta_{F}^{\prime}$ be $\Delta_{F}$ where the off-diagonal elements of the rows defined by the positions of $I_{\partial M}$ set to 0 and corresponding elements on the main diagonal set to 1 . The refined coordinates $\left(\boldsymbol{x}^{*^{\prime}}, \boldsymbol{y}^{*^{\prime}}\right)$ of the vertices of $M_{F}$ in the final flat representation can be found by solving the following system of linear equations:

$$
\begin{aligned}
& \boldsymbol{\Delta}_{\boldsymbol{F}}^{\prime} \boldsymbol{x}^{*^{\prime}}=\boldsymbol{b}_{x}^{\prime} \\
& \boldsymbol{\Delta}_{\boldsymbol{F}}^{\prime} \boldsymbol{y}^{*^{\prime}}=\boldsymbol{b}_{y}^{\prime}
\end{aligned}
$$




\section{Experiments and Results}

We used the proposed flattening method to visualise atrial wall thickness (AWT) of both left and right atria. Inspecting heterogeneity of AWT may be useful to improve the understanding of the mechanisms underlying atrial fibrillation and to improve radio-frequency ablation treatment planing. Detailed endocardial and epicardial surface meshes were first obtained by segmenting the atrial wall from the MRIs corresponding to an ex vivo human atria and then, AWT was measured as explained in [18]. Wall thickness measurements were projected onto the endocardial atrial meshes which were afterwards flattened with our method. As shown in Fig. 2, atrial unfolding favours fast inspection of AWT measurements: regarding the LA, it can be seen that the inter-atrial septal wall and the posterior wall were thinner than the left lateral and anterior walls; a thin inter-atrial wall was also observed in the RA, where one of the thickest parts was the crista terminalis $(\mathrm{CT})$.

The proposed flattening method was also tested using several manually segmented LA from the 2018 Atrial Segmentation Challenge ${ }^{5}$, and corresponding RA segmentations that were automatically obtained applying a multi-atlas whole-heart segmentation technique [19,20]. Triangular meshes were then built using the marching cubes algorithm. Voxel intensities from LGE-CMR images were mapped onto the obtained RA and LA surface mesh using the maximum intensity projection (MIP) technique: images were sampled along the normals on both sides of the surface mesh, assigning the maximum intensity value to the corresponding vertex on the LA mesh. The depth of the sampling was set to 3 $\mathrm{mm}$. Then, the LA shapes were standardised by only keeping their main cavity after semi-automatically cutting the PVs, the LAA and the MV. This cutting process requires to manually place 5 seeds near the ending points of the PVs and the LAA. The reader is referred to [16] for more details on this method. Regarding the RA, cuts corresponding to the TV, SVC and IVC boundaries were manually applied. Several examples of 3D left and right atrial surfaces with projected LGE-CMR image intensity, and corresponding flattenings can be seen in Fig. 3.

Additional examples and experiments including distortion analysis, sensitivity to seed points selection, and a detailed comparison with the SUM [17] can be found in [10].

\section{Discussion and Conclusions}

We have presented a method to unfold both the left and right atrium and depict it in an intuitive, standardised, two-dimensional map. Contrary to the state of the art method for LA standardised representation, the SUM [17], our method does not depend on registration techniques being therefore faster and not affected by potential registration errors. Additionally, our method does not lose any information from the initial 3D surface, i.e. all points are depicted in the

\footnotetext{
${ }^{5}$ http://atriaseg2018.cardiacatlas.org/
} 


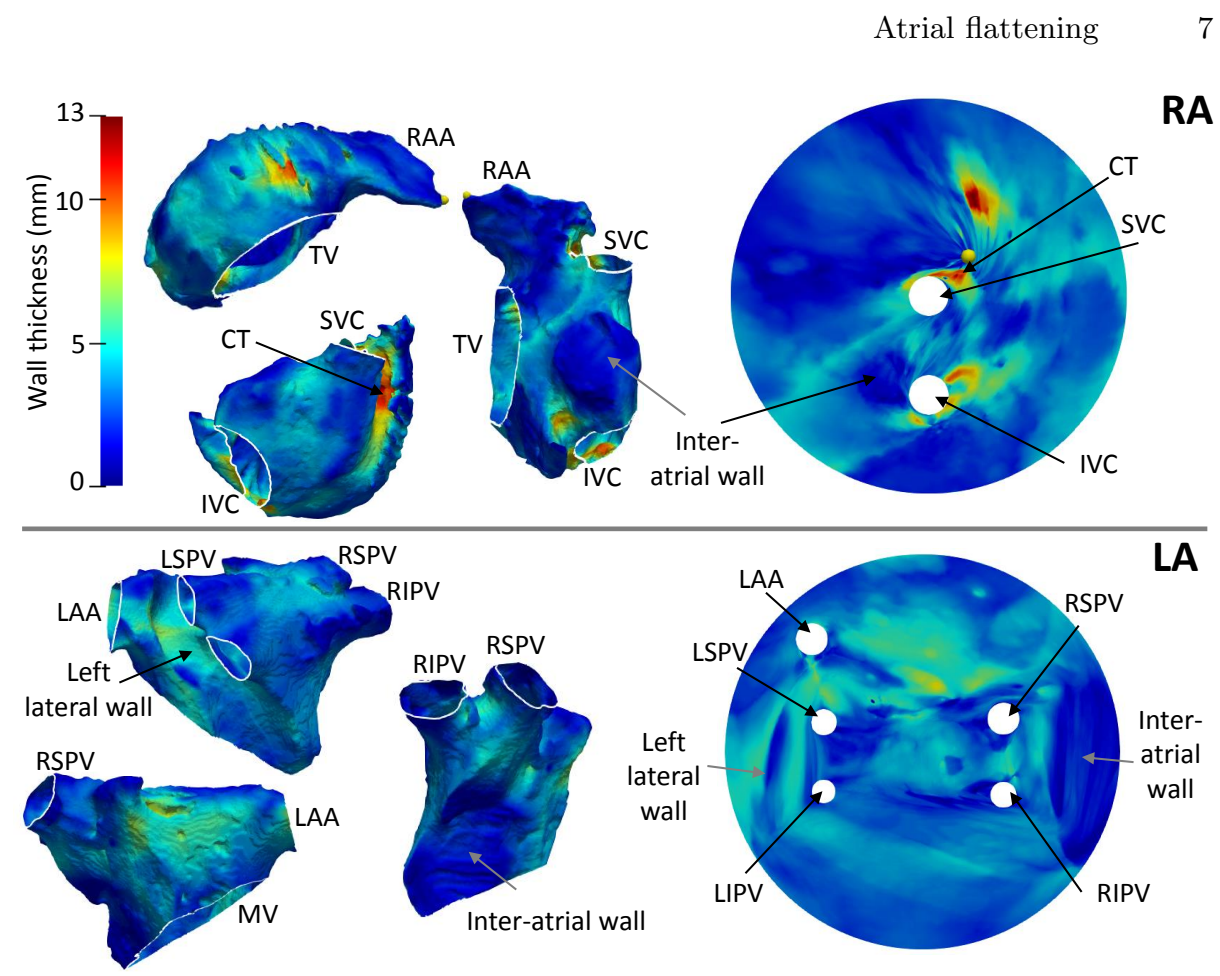

Fig. 2. Right (top) and left (bottom) atrial wall thickness measurements projected onto the endorcardial surface meshes and corresponding $2 \mathrm{D}$ maps. LAA/RAA = left/right atrial appendage; TV = tricuspid valve; $\mathrm{SVC} / \mathrm{IVC}=$ superior/inferior vena cava; $\mathrm{CT}$ $=$ crista terminalis; LSPV $=$ left superior PV; LIPV $=$ left inferior PV; RSPV $=$ right superior PV; RIPV = right inferior $\mathrm{PV} ; \mathrm{MV}=$ mitral valve.

2D map, while SUM needs to interpolate data when mapping the information from the arbitrary 3D surface to the template. Our algorithm is however highly influenced by the initial manual seed placement step, especially regarding the seeds placed on the MV contour. The seed points and inter-seed paths define the constraints used in the flattening, which is the key part of the method. Automatically computing the constraints, i.e. automatically dividing the LA is challenging due to, for example, bulges in the LA and obliqueness of the MV plane. Nonetheless, since the process of seed placement and unfolding is almost real time it can be repeated several times until a satisfactory result is obtained.

Potential clinical applications of our method include the analysis of the temporal evolution of some parameter (e.g. pre- and post-ablation fibrosis extent) from the same patient; the comparison and correlation of different features from the same patient (e.g. gadolinium enhancement from LGE-CMR data and endocardial voltage from electroanatomical maps); the comparison of the same feature in different patients (e.g. regional or global fibrosis extent [1]), etc. The code will be made publicly available. 


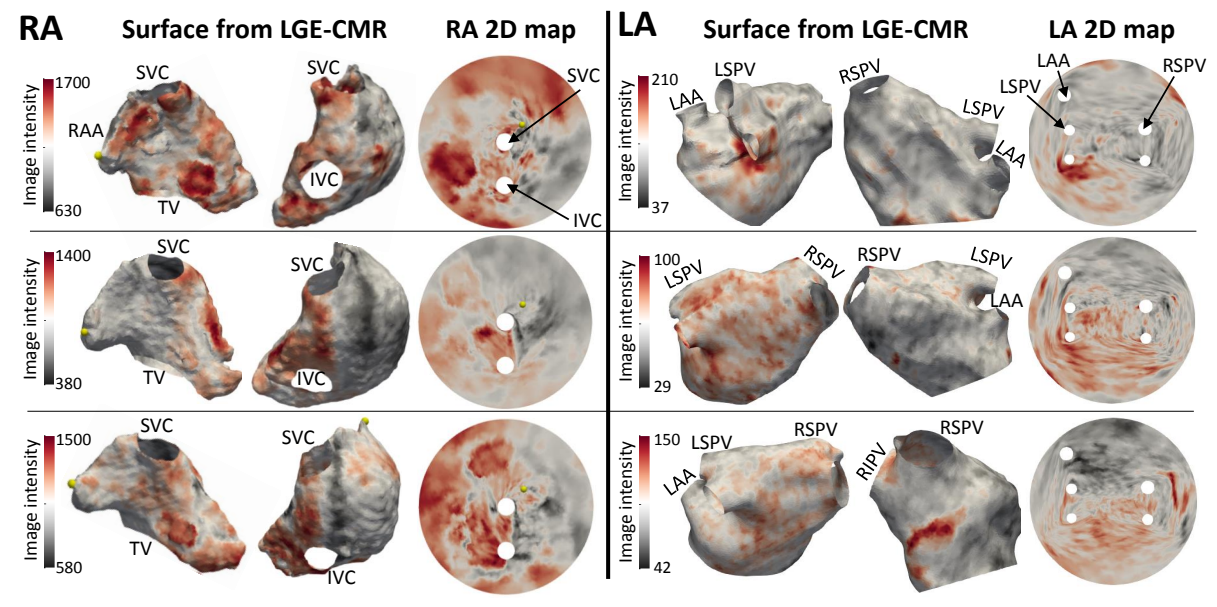

Fig. 3. Three examples of RA (left) and three examples of LA (right) surfaces with projected late gadolinium enhanced magnetic resonance imaging (LGE-CMR) intensities and corresponding 2D maps. LGE-CMR signal intensity is shown in arbitrary units, without any normalization [4]. LAA/RAA = left/right atrial appendage; TV = tricuspid valve; $\mathrm{SVC} / \mathrm{IVC}=$ superior/inferior vena cava; LSPV = left superior PV; $\mathrm{LIPV}=$ left inferior $\mathrm{PV} ; \mathrm{RSPV}=$ right superior $\mathrm{PV} ; \mathrm{RIPV}=$ right inferior $\mathrm{PV}$.

\section{Acknowledgments}

This study was partially funded by the Spanish Ministry of Economy and Competitiveness (DPI2015-71640-R), by the "Fundació La Marató de TV3" (n ${ }^{\circ}$ 20154031) and by European Union Horizon 2020 Programme for Research and Innovation, under grant agreement No. 642676 (CardioFunXion).

\section{References}

1. Benito, E.M., Cabanelas, N., Nuñez-Garcia, M., Alarcón, F., Ventura, F.I., Rosa, M., Soto-Iglesias, D., Guasch, E., Prat-Gonzalez, S., Perea, R.J., et al.: Preferential regional distribution of atrial fibrosis in posterior wall around left inferior pulmonary vein as identified by late gadolinium enhancement cardiac magnetic resonance in patients with atrial fibrillation. EP Europace (2018)

2. Cerqueira, M.D., Weissman, N.J., Dilsizian, V., Jacobs, A.K., Kaul, S., Laskey, W.K., Pennell, D.J., Rumberger, J.A., Ryan, T., Verani, M.S., et al.: Standardized myocardial segmentation and nomenclature for tomographic imaging of the heart: a statement for healthcare professionals from the cardiac imaging committee of the council on clinical cardiology of the american heart association. Circulation 105(4), 539-542 (2002)

3. Karim, R., Ma, Y., Jang, M., Housden, R.J., Williams, S.E., Chen, Z., Ataollahi, A., Althoefer, K., Rinaldi, C.A., Razavi, R., et al.: Surface flattening of the human left atrium and proof-of-concept clinical applications. Computerized Medical Imaging and Graphics 38(4), 251-266 (2014) 
4. Khurram, I.M., Beinart, R., Zipunnikov, V., Dewire, J., Yarmohammadi, H., Sasaki, T., Spragg, D.D., Marine, J.E., Berger, R.D., Halperin, H.R., et al.: Magnetic resonance image intensity ratio, a normalized measure to enable interpatient comparability of left atrial fibrosis. Heart rhythm 11(1), 85-92 (2014)

5. Kreiser, J., Meuschke, M., Mistelbauer, G., Preim, B., Ropinski, T.: A Survey of Flattening-Based Medical Visualization Techniques. In: Computer Graphics Forum. vol. 37. No. 3, pp. 597-624 (2018)

6. Lévy, B., Petitjean, S., Ray, N., Maillot, J.: Least squares conformal maps for automatic texture atlas generation. In: ACM transactions on graphics (TOG). vol. 21, No. 3., pp. 362-371 (2002)

7. Liepa, P.: Filling holes in meshes. In: Proceedings of the 2003 Eurographics/ACM SIGGRAPH symposium on Geometry processing. pp. 200-205. Eurographics Association (2003)

8. Ma, Y., Karim, R., Housden, R.J., Gijsbers, G., Bullens, R., Rinaldi, C.A., Razavi, R., Schaeffter, T., Rhode, K.S.: Cardiac unfold: a novel technique for image-guided cardiac catheterization procedures. In: International Conference on Information Processing in Computer-Assisted Interventions. pp. 104-114 (2012)

9. Mitchell, J.S., Mount, D.M., Papadimitriou, C.H.: The discrete geodesic problem. SIAM Journal on Computing 16(4), 647-668 (1987)

10. Núñez García, M.: Left atrial parameterisation and multi-modal data analysis: application to atrial fibrillation. Ph.D. thesis, Universitat Pompeu Fabra (2018)

11. Paun, B., Bijnens, B., Iles, T., Iaizzo, P.A., Butakoff, C.: Patient independent representation of the detailed cardiac ventricular anatomy. Medical image analysis 35, 270-287 (2017)

12. Pinkall, U., Polthier, K.: Computing discrete minimal surfaces and their conjugates. Experimental mathematics 2(1), 15-36 (1993)

13. Prasanna, L., Praveena, R., AS, D., Kumar, M.: Variations in the pulmonary venous ostium in the left atrium and its clinical importance. Journal of clinical and diagnostic research 8(2), 10 (2014)

14. Roney, C.H., Pashaei, A., Meo, M., Dubois, R., Boyle, P.M., Trayanova, N.A., Cochet, H., Niederer, S.A., Vigmond, E.J.: Universal atrial coordinates applied to visualisation, registration and construction of patient specific meshes. arXiv preprint arXiv:1810.06630 (2018)

15. Soto-Iglesias, D., Butakoff, C., Andreu, D., Fernández-Armenta, J., Berruezo, A., Camara, O.: Integration of electro-anatomical and imaging data of the left ventricle: an evaluation framework. Medical image analysis 32, 131-144 (2016)

16. Tobon-Gomez, C., Geers, A.J., Peters, J., Weese, J., Pinto, K., Karim, R., Ammar, M., Daoudi, A., Margeta, J., Sandoval, Z., et al.: Benchmark for algorithms segmenting the left atrium from 3D CT and MRI datasets. IEEE transactions on medical imaging 34(7), 1460-1473 (2015)

17. Williams, S.E., Tobon-Gomez, C., Zuluaga, M.A., Chubb, H., Butakoff, C., Karim, R., Ahmed, E., Camara, O., Rhode, K.S.: Standardized unfold mapping: a technique to permit left atrial regional data display and analysis. Journal of Interventional Cardiac Electrophysiology 50(1), 125-131 (2017)

18. Zhao, J., Hansen, B.J., Wang, Y., Csepe, T.A., Sul, L.V., Tang, A., Yuan, Y., Li, N., Bratasz, A., Powell, K.A., et al.: Three-dimensional integrated functional, structural, and computational mapping to define the structural fingerprints of heartspecific atrial fibrillation drivers in human heart ex vivo. Journal of the American Heart Association 6(8), e005922 (2017) 
19. Zhuang, X., Bai, W., Song, J., Zhan, S., Qian, X., Shi, W., Lian, Y., Rueckert, D.: Multiatlas whole heart segmentation of CT data using conditional entropy for atlas ranking and selection. Medical physics 42(7), 3822-3833 (2015)

20. Zhuang, X., Shen, J.: Multi-scale patch and multi-modality atlases for whole heart segmentation of MRI. Medical image analysis 31, 77-87 (2016) 